\title{
Robotic Resection of a Large Degenerating Leiomyoma
}

\author{
Cari Eckman ${ }^{1}$, Andrea L Buras ${ }^{1,2 *}$, Daniel K Jeong ${ }^{3}$, Ardeshir Hakam ${ }^{4}$ and Hye Sook Chon ${ }^{2}$
}

${ }^{1}$ Department of Obstetrics and Gynecology, University of South Florida, Florida, USA

${ }^{2}$ Department of Gynecologic Oncology, H Lee Moffitt Cancer Center and Research Institute, Florida, USA

${ }^{3}$ Department of Diagnostic Imaging and Interventional Radiology, $H$ Lee Moffitt Cancer Center and Research Institute, Florida, USA

${ }^{4}$ Department of Anatomic Pathology, H Lee Moffitt Cancer Center and Research Institute, Florida, USA

*Corresponding author: Andrea L Buras, Department of Obstetrics and Gynecology, $H$ Lee Moffitt Cancer Center and Research Institute, University of South Florida, FL, 12902 USF Magnolia Drive, Tampa, FL 33612, USA, Tel: 248-933-4942, Fax: 813-449-8002

\section{Background}

Uterine leiomyomas are benign myometrial smooth muscle neoplasms. The cumulative incidence of uterine leiomyomata has been found to be as high as $70 \%$ in white women and above $80 \%$ in black women [1]. Leiomyomas frequently undergo coagulative necrosis with smooth muscle being replaced by various degenerative substances; forms of degeneration include hyaline, calcific, cystic, fatty, hemorrhagic or red, and myxoid. Cystic degeneration, occurring in $4 \%$ of all degenerated fibroids, typically appears as multiple hypoechoic or anechoic areas that are irregular and smooth walled [2]. Magnetic resonance imaging (MRI) is the most accurate imaging modality for leiomyomas [3]. Non-degenerated leiomyomas appear as homogenous well-circumscribed masses on MRI. Cystic degeneration of fibroids on MRI demonstrates high signal intensity on $\mathrm{T} 2$ images and do not enhance, which may appear similar to leiomyosarcomas, ovarian malignancies, or other retroperitoneal masses such as myxoid liposarcoma, neurofibroma, schwannoma, or lymphangioma which also have cystic characteristics $[3,4]$. Additionally, approximately $11.8 \%$ of fibroids with cystic degeneration extend into the retroperitoneal space [5].

A review of the literature demonstrates 19 cases of degenerated fibroids that were misdiagnosed as an ovarian malignancy on imaging prior to surgery [6-9].
Of these cases reported, only one mass was removed laparoscopically through a $2 \mathrm{~cm}$ port at the umbilicus [9]. This case report details how a giant degenerative fibroid diagnosed as a suspected ovarian neoplasm with possible retroperitoneal extension was successfully managed utilizing a minimally invasive approach.

\section{Case Presentation}

A 56-year-old African American, multiparous, postmenopausal woman presented with a cystic left adnexal mass found incidentally on her yearly transvaginal ultrasound to monitor fibroids. She had a known history of uterine leiomyomas for which she had not required treatment. Her only previous abdominal surgery was a tubal ligation.

Pelvic examination demonstrated a mobile, nontender uterus with a large palpable left adnexal mass that was smooth and mobile. A transvaginal ultrasound demonstrated a heterogenous fibroid uterus. The ovaries were unable to be visualized. Bilateral soft tissue adnexal masses were present with an undetermined nature and no vascularity. Pelvic MRI demonstrated numerous uterine fibroids as well as a large, heterogeneously $\mathrm{T} 2$ hyperintense lesion within the left pelvis measuring approximately $10.5 \times 17.5 \mathrm{~cm}$. This mass was found to be situated posteriorly into the presacral space and extended anteriorly to the anterior lower abdominal wall. The left anterior aspect of this lesion 


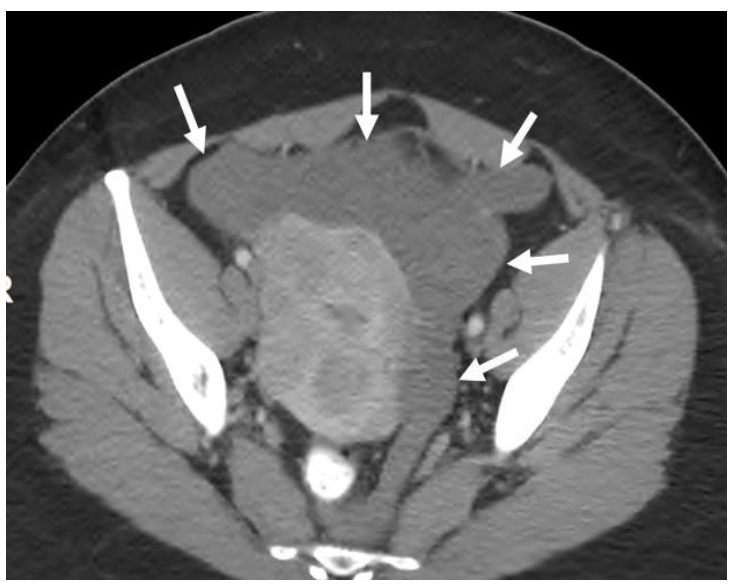

A

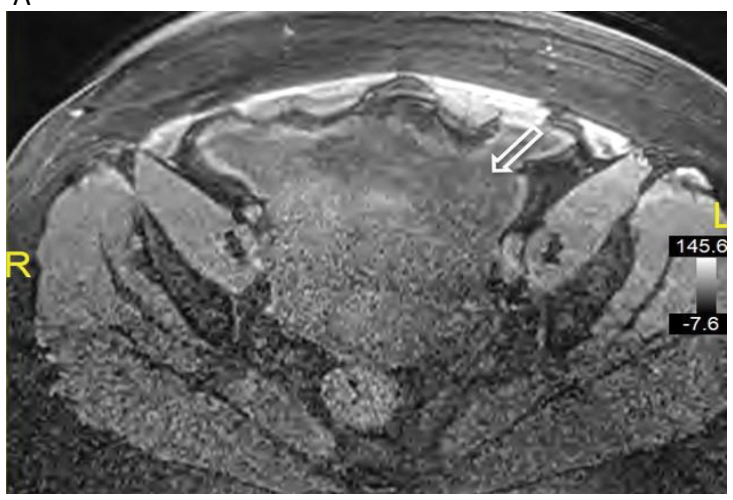

C

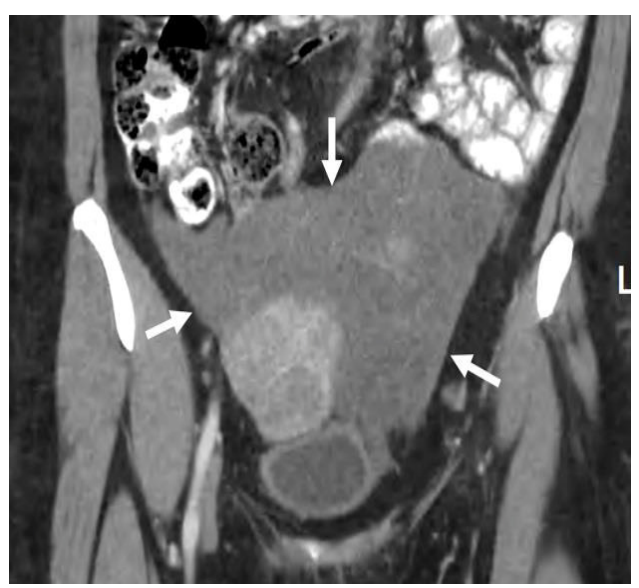

B

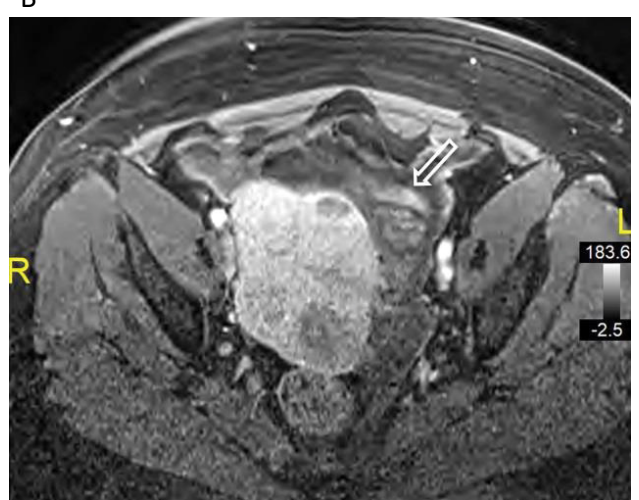

D

Figure 1: A) Axial post contrast CT through the pelvis demonstrates an amorphous low-density mass (white arrows) surrounding enhancing fibroids within the uterus. The mass extends from the anterior pelvic wall region through the left adnexal region to the presacral space; B) Coronal reconstructed post contrast CT demonstrates the heterogenous nature of the mass (white arrows); C) Axial pre-contrast T1 weighted fat saturated MR image demonstrates a heterogenous T1 signal hypointense mass; D) Axial post-contrast T1 weighted fat saturated MR image demonstrates focal enhancing nodules within the amorphous hypointense mass (open arrow).

appeared to be intimately associated with the left ovary. There was also $\mathrm{T} 2$ hyperintense material extending throughout the anterior pelvis both left and right. The margins of this collection demonstrated a thin rim of enhancement and there appeared to be a heterogenous, relatively nodular enhancement within the left anterior lower pelvic component (Figure 1 and Figure 2). Subsequent computed tomography (CT) scan of the abdomen and pelvis with and without contrast demonstrated a large complex cystic mass extending throughout the pelvis measuring $17.1 \times 15.5 \times 11.9 \mathrm{~cm}$; it was suspected to be arising from the left adnexa (Figure 1 and Figure 2). Tumor markers including CA 125, CEA and CA 19-9 were normal.

Surgical intervention was planned due to imaging findings. Upon laparoscopic abdominopelvic surveillance, no ascites was noted; Liver, stomach, omentum, and diaphragm all appeared normal. The uterus was enlarged with multiple fibroids. Right fallopian tube and ovary were normal. An elongated cystic, loose, soft mass was noted in the left pelvis extending to the anterior abdominal wall. A normal appearing left ovary and fallopian tube connected to the mass posteriorly. The mass extended in the retroperitoneal space along the left sidewall to the para-vesicle space. The mass was meticulously dissected from the rectosigmoid colon, left ureter and left sidewall to the para-vesicle space, completing dissection off the bladder. The remainder of the bilateral salpingo-oophorectomy and hysterectomy were performed in the usual fashion. Uterus, fallopian tubes, ovaries and mass were removed from the abdomen via colpotomy. The patient did well postoperatively and was discharged home the same day without complication.

Specimen of pelvic mass, uterus, cervix, fallopian tubes, and ovaries weighed $1072.2 \mathrm{~g}$ (Figure 3A). The collapsed cystic mass measures $12.5 \times 12.5 \times$ $3.0 \mathrm{~cm}$ and has a pink, glistening, soft, ragged, partially ruptured external surface. Sectioning of the cystic structure reveals tan-yellow, mucoid cut surfaces, with no discrete firm masses or papillary excrescences grossly identified. The endometrial cavity is normal appearing with no involvement with the cystic structure. Final pathology was consistent with leiomyoma with degenerative changes (Figure 3B and Figure 3 C).

\section{Discussion}

Uterine leiomyomas are common benign smooth 

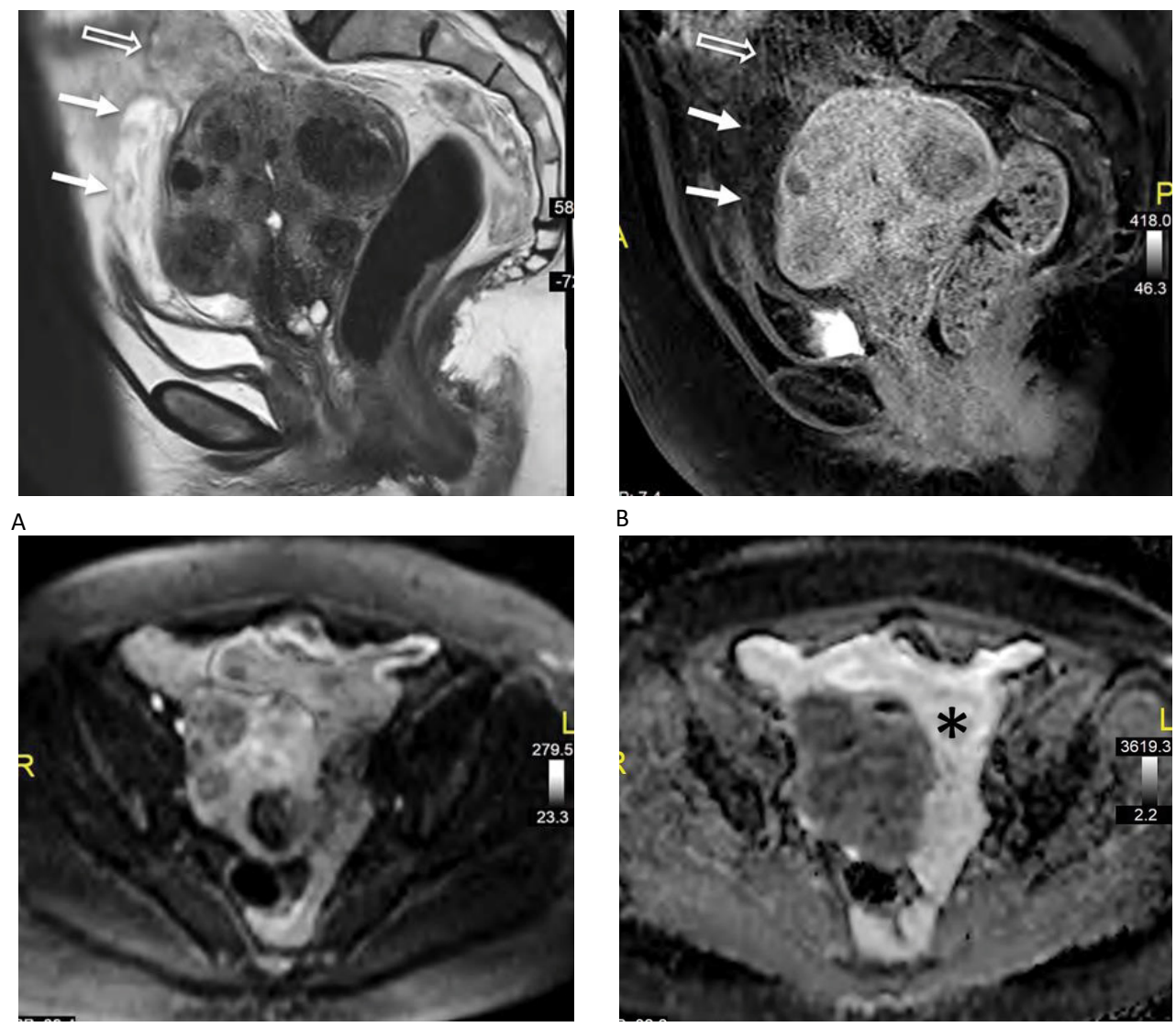

C

$\mathrm{D}$

Figure 2: A) Sagittal T2 weighted MR image demonstrates a fibroid uterus with heterogenous abnormal T2 hyperintense signal anterior and superior to the uterus. There is a T2 hyperintense region (white arrows) and a more heterogenous T2 hypointense region (open arrow) within the mass; B) Sagittal T1 weighted post-contrast MR image demonstrates hypoenhancement within the lower anterior component (white arrows) with subtle enhancement in the superior component (open arrow); C) Axial diffusion weighted b800 MR image shows heterogenous signal within the amorphous mass but without frank restricted diffusion; D) Axial apparent diffusion coefficient map demonstrates no frank restricted diffusion. $A D C$ value within the amorphous left adnexal region near the enhancing nodule ( $\left.{ }^{*}\right)$ was $2.39 \times 10-3 \mathrm{~mm}^{2} / \mathrm{s}$ suggesting no restriction.

A

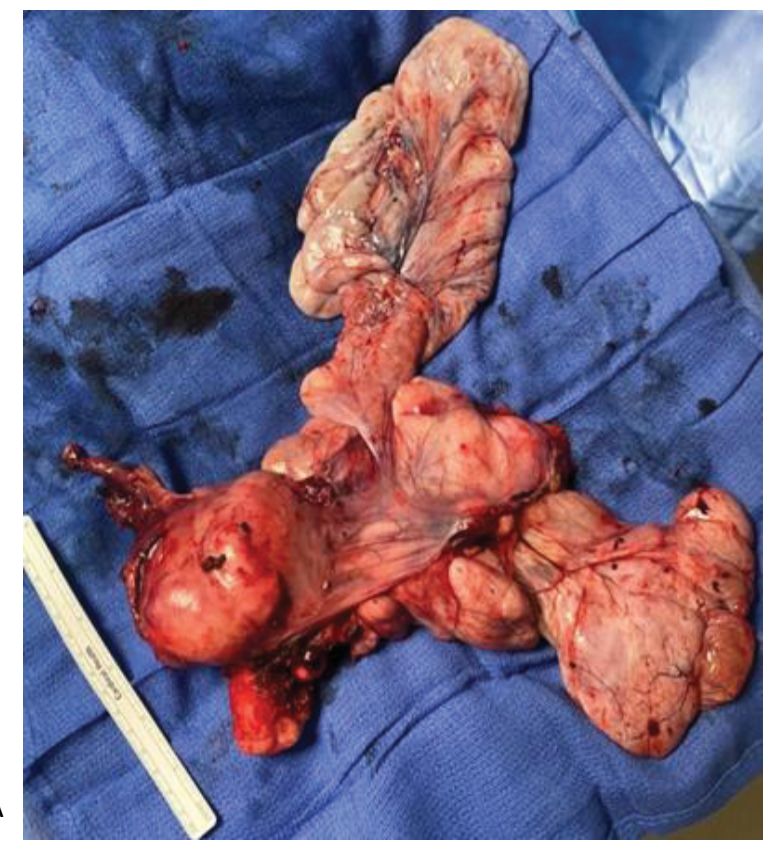

B

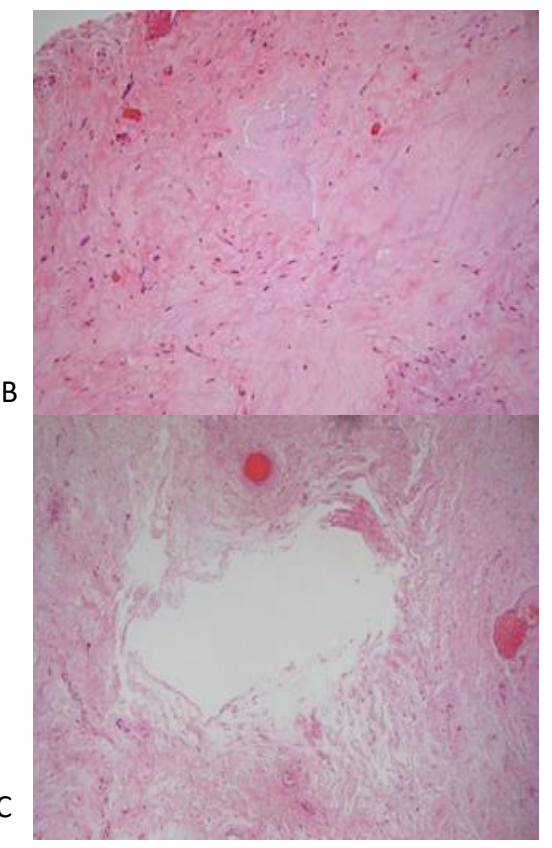

Figure 3: A) Surgical specimen including uterus (white arrow), cervix (black arrow), fallopian tubes, ovaries, and large cystic structure $\left({ }^{*}\right)$, removed intact; B) 10X power demonstrating edematous changes of leiomyoma; C) $5 \mathrm{X}$ power demonstrating cystic changes seen in degenerating leiomyoma. 
muscle neoplasms; frequently they are asymptomatic, though they can cause pelvic pain, pressure, abnormal uterine bleeding, and several other symptoms that affect quality of life. While the uterus is the most common location for leiomyoma, extra-uterine leiomyoma may also occur, within the broad ligament, ovary, and vulva, for example, or more diffusely as benign metastasizing leiomyoma or peritoneal leiomyomatosis. They may outgrow their blood supply and undergo one of several forms of degeneration. These variable features can lead to a diagnostic dilemma.

MRI is the most accurate imaging technique for identification and characterization of leiomyomas; however, choice of imaging modality should be guided by the patient's specific clinical scenario and differential diagnosis. In our case, initial pelvic US findings warranted additional imaging and an MRI was performed. MRI demonstrated a large, heterogeneously T2 hyperintense lesion within the left pelvis with a thin rim of enhancement. This is consistent with but not specific for degenerating leiomyoma. Additional imaging with CT scan demonstrated pelvic mass suspected to be arising from the ovary, however, no other signs of malignancy were identified. Additionally, all tumor markers were normal.

Uterine leiomyomas are typically able to be diagnosed with various imaging modalities, but cystic degeneration makes diagnosis more challenging especially when located in the retroperitoneum. There are many tumors with cystic characteristics that can appear similar to a degenerative leiomyoma including myxoid liposarcoma, schwannoma, and neurofibroma which all demonstrate cystic masses with solid tumor enhancement on imaging [4]. In a literature review of 37 cases of retroperitoneal leiomyoma, diagnosis was inconclusive using imaging and operative management was pursued in all but one case [5].

Given enlarging pelvic mass, suspicious for ovarian neoplasm but low suspicion for malignancy, decision was made to proceed with surgical removal. Frequently in these cases, due to large size and concern for possible ovarian malignancy, such patients are managed with laparotomy. Our case demonstrates the safety and feasibility of a minimally invasive approach in the management of degenerating fibroid. Given the intraoperative findings, it is likely that this lesion arose from a broad ligament leiomyoma, expanding into the retroperitoneal space with its degeneration. It is notable that degenerating leiomyoma with cystic characteristics are often pliable and as long as careful dissection can be completed without rupture, these can be safely removed vaginally with or without the assistance of a bag to prevent intra-abdominal rupture of the mass. We were able to completely excise the degenerating leiomyoma, without rupture robotically and remove it without extending any abdominal incisions. Without a clear indication for laparotomy, minimally invasive surgery can be used to characterize and manage pelvic and retroperitoneal masses, providing the patient with the known benefits of minimally invasive procedure.

\section{Conflict of Interest Statement}

The authors have no conflicts of interest to disclose.

\section{Author Contributions}

Cari Eckman: Writing - original draft; Andrea Buras: Writing - original draft, writing - review and editing, Visualization; Daniel K Jeong: Resources, Writing review and editing; Ardeshir Hakam: Resources, Writing - review and editing; Hye Sook Chon: Writing - review and editing, Supervision.

\section{References}

1. Wise LA, Laughlin-Tommaso SK (2016) Epidemiology of uterine fibroids: From menarche to menopause. Clin Obstet Gynecol 59: 2-24.

2. Persaud V, Arjoon PD (1970) Uterine leiomyoma. Incidence of degenerative change and a correlation of associated symptoms. Obstet Gynecol 35: 432-436.

3. Murase E, Siegelman ES, Outwater EK, Perez-Jaffe LA, Tureck RW (1999) Uterine leiomyomas: Histopathologic features, MR imaging findings, differential diagnosis, and treatment. Radiographics 19: 1179-1197.

4. Rajiah P, Sinha R, Cuevas C, Dubinsky TJ, Bush WH Jr, et al. (2011) Imaging of uncommon retroperitoneal masses. Radiographics 31: 949-976.

5. Poliquin V, Victory R, Vilos GA (2008) Epidemiology, presentation, and management of retroperitoneal leiomyomata: Systematic literature review and case report. J Minim Invasive Gynecol 15: 152-160.

6. Sharma P, Zaheer S, Yadav AK, Mandal AK (2016) Massive broad ligament cellular leiomyoma with cystic change: $A$ diagnostic dilemma. J Clin Diagn Res 10: ED01-ED02.

7. Yorita K, Tanaka Y, Hirano K, Kai Y, Arii K, et al. (2016) A subserosal, pedunculated, multilocular uterine leiomyoma with ovarian tumor-like morphology and histological architecture of adenomatoid tumors: A case report and review of the literature. J Med Case Rep 10: 352.

8. Jha S, Singh A, Singh S, Murmu S (2020) Huge broad ligament leiomyoma with cystic degeneration: A diagnostic and surgical challenge. J Obstet Gynaecol Res 46: 791794.

9. Walker C, Banning K, Ritchie C, Kliethermes C (2020) Laparoscopic management of a degenerating cystic leiomyoma imitating an ovarian cyst: A case report. Case Rep Womens Health 27: e00205.

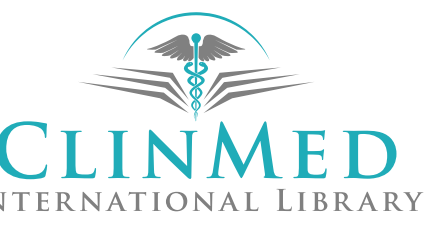

